\title{
APLICAÇÃO DA CURVA ABC NO GERENCIAMENTO DE ESTOQUE EM UMA EMPRESA DE CONSTRUÇÃO CIVIL
}

\section{APPLICATION OF THE ABC CURVE IN STOCK MANAGEMENT IN A CIVIL CONSTRUCTION COMPANY}

\author{
Rafaela Ribeiro Siqueira ${ }^{1 *}$, Iasmine Aléxia de Aquino Melo ${ }^{2}$, \& Ricardo Bentes Kato ${ }^{3}$ \\ 123 Universidade Federal do Pará, UFPA, Brasil. \\ 1* raphaela_rs15@hotmail.com ${ }^{2} \underline{\text { iasminealexia@gmail.com }}^{3} \underline{\text { ricardokato@ufpa.br }}$
}

\section{ARTIGO INFO.}

\section{Recebido em: 16.09.2021}

Aprovado em: 11.11.2021

Disponibilizado em: 29.11.2021

Palavras-Chave:

Gestão de estoques; Ferramenta; Classificação ABC.

KEYWORDS:

Inventory management; Tool; ABC classification.

*Autor Correspondente: Siqueira, R. R.

\section{RESUMO}

O progressivo aumento da competitividade, as organizações buscam formas de melhorar seus processos com a finalidade de reduzir custos. Deste modo, é recomendável fazer um bom planejamento que permita uma tomada de decisão para o gerenciamento de estoque. Neste sentido, aplica-se a curva $\mathrm{ABC}$ como uma ferramenta de apoio para o controle de estoque, permitindo aos gestores uma visão detalhada dos produtos. Portanto, a presente pesquisa tem como objetivo discutir a aplicação da ferramenta $\mathrm{ABC}$ em uma empresa que atua no segmento da construção civil, situada na cidade de Marabá-PA. Foram realizadas visitas na empresa para entender todo o processo de gestão de estoque, assim como entrevistas não estruturadas com o gerente do setor e o profissional responsável pelo almoxarifado. Para tratar os problemas encontrados foi utilizado a ferramenta de classificação ABC, para definir a priorização das ações gerenciais sobre os itens em estoque e assim saber quais os itens de maior relevância. Logo, os resultados obtidos vão de contrariedade da teoria proposta por Vilfredo Pareto, de estoques no sistema 80/20, e que itens da classe A devem possuir estoques baixos, enquanto que itens da classe $\mathrm{C}$ devem ter estoques altos. Por meio intermédio da ferramenta, encontrou se os seguintes resultados classe $\mathrm{A}$, possuem tamanho de estoque alto, com 11.352, Classe B dos 19.552 materiais presentes no almoxarifado, os itens classificados como C correspondem a apenas 3.237 dos materiais em estoque. Essa ferramenta aplicada trouxe reais e concretas informações do processo de gestão de estoques da empresa em questão, dando assim suporte ao entendimento do estoque para os gestores.

\begin{abstract}
The progressive increase in competitiveness, organizations are looking for ways to improve their processes in order to reduce costs. Thus, it is recommended to do a good planning that allows decision making for stock management. In this sense, the ABC curve is applied as a support tool for stock control, allowing managers to have a detailed view of the products. Therefore, this research aims to discuss the application of the $A B C$ tool in a company that operates in the civil construction sector, located in the city of Marabá-PA. Visits were carried out at the company to understand the entire inventory management process, as well as unstructured interviews with the sector manager and the professional responsible for the warehouse. To deal with the problems found, the ABC classification tool was used, to define the prioritization of managerial actions on the items in stock and thus to know which items are of greater relevance. Therefore, the results obtained go against the theory proposed by Vilfredo Pareto, of stocks in the 80/20 system, and that class $A$ items must have low stocks, while class $C$ items must have high stocks. Through the tool, it was found if the following class A results have a high stock size, with 11,352, Class B of the 19,552 materials present in the warehouse, the items classified as $C$ correspond to only 3,237 of the materials in stock. This applied tool brought real and concrete information about the company's stock management process, thus supporting the understanding of stock for managers
\end{abstract}




\section{INTRODUÇÃO}

A indústria da construção civil tem um importante peso sobre a economia brasileira. Na última década, foi considerada como um dos setores mais dinâmicos e responsável pela maior parte do desenvolvimento econômico na última década, no Brasil. Em 2019, o setor da construção civil teve uma participação de 3,7\% do Produto Interno Bruto (PIB) brasileiro, segundo (IBGE, 2019).

Essa significativa participação na economia nacional, possibilitou que por muito tempo o setor técnico-estrutural recebesse mais atenção que a área de administração de materiais, mesmo que estes representem grande parte dos recursos de uma construção (Szajubok, Alencar, \& Almeida, 2006). Deste modo, o setor de materiais é uma das áreas que mais sofre com a falta de gestão.

O almoxarifado é o ambiente onde é realizado o armazenamento dos materiais que serão utilizados em todas as etapas de construção de um empreendimento na indústria da construção civil. Na prática, o almoxarifado deverá ser organizado de forma a melhorar o trabalho, diminuir os custos, racionalizar o espaço, possibilitar rápida identificação e facilitar a retirada dos materiais (Martins e Laugeni, 2009). No entanto, nesse ambiente é comum encontrar problemas relacionados com a má localização dos estoques, armazenagem inadequada, incompatibilidade do estoque físico com dados do inventário e erros gerados no recebimento (Dias, 2010).

A partir desse cenário, surge a administração de estoques com o objetivo de auxiliar na gestão e no controle dos materiais necessários para o funcionamento das organizações, de forma a otimizar o desempenho do almoxarifado de uma empresa. Em vista disso, este assunto tem sido objeto de muitos estudos mencionados na literatura, como os modelos estocásticos para a determinação da quantidade de pedido, técnicas de projeção de demanda e técnicas $\mathrm{ABC}$ (Szajubok, Alencar, \& Almeida, 2006). Sendo que, este último engloba todos os itens que compõe o estoque e os classifica em: importantes, intermediários e os menos importantes (Viana, 2009).

Diante desse contexto, foi realizada uma análise para otimização de estoques na construção civil por meio do modelo de gestão da Curva $\mathrm{ABC}$, aplicando-o através de um estudo de caso em uma empresa que atua neste segmento localizada na cidade de Marabá-PA. Busca-se, a partir deste estudo identificar o grau de importância dos itens com base em sua relevância no empreendimento em questão e aprofundar as discussões acerca da necessidade de uma gestão de estoques nas empresas.

\section{REFERENCIAL TEÓRICO}

A gestão de estoque é um dos pontos críticos da indústria da construção e deve ser constantemente monitorada. Nesse sentido, Martins e Laugeni (2009) apontam que a ausência de controle de entrada e saída de materiais pode levar a falta de estoque, resultando na paralisação da produção e consequentemente na redução dos retornos financeiros. Por outro lado, de acordo com Dias (2010) grandes estoques acarretam maiores custos, pois o capital investido na compra dos materiais fica parado durante um período mais longo (Dias, 2010). Portanto, a gestão de estoque funciona de forma a garantir que os estoques não sejam nem altos e nem baixos. Para tanto, os administradores devem colocar em prática o ciclo de administração de materiais, apresentado na Figura 1, proposto por Martins e Laugeni (2009). 

empresa de construção civil. Brazilian Journal of Production Engineering, 7(5), 145-155.

Figura 1. Ciclo de administração de materiais

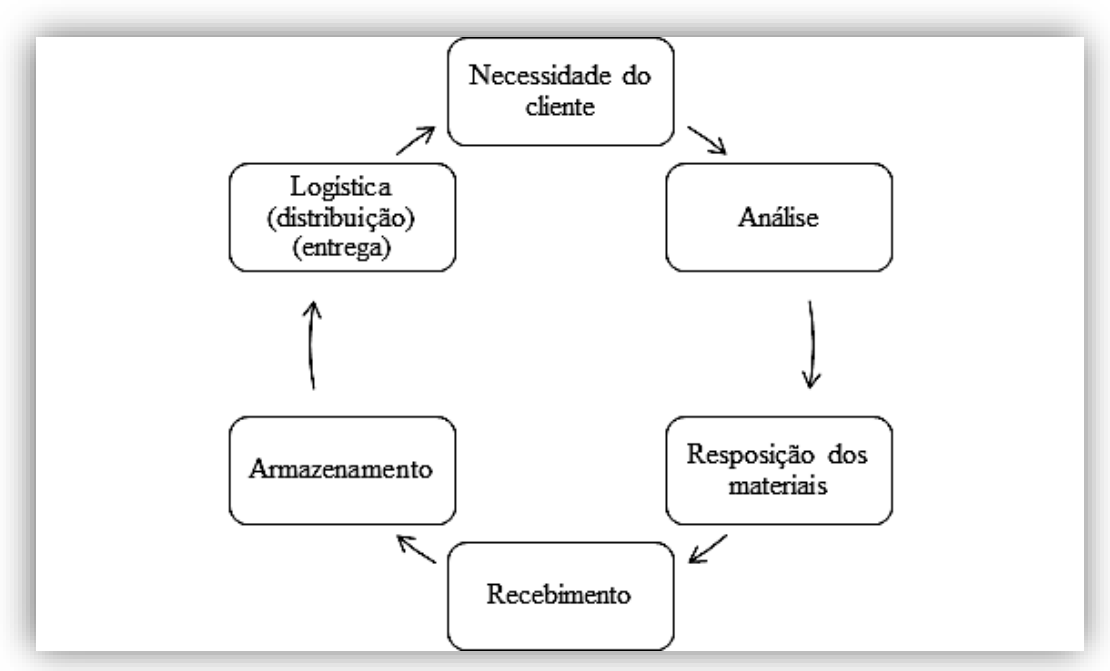

Fonte: Adaptado de Martins e Laugeni (2009)

De acordo com Martins e Laugeni (2009) o ciclo de administração dos materiais inicia-se com as necessidades dos clientes internos e externos. Essas solicitações dos clientes são analisadas pelas empresas, de modo a avaliar a capacidade de atendimentos a partir dos estoques existentes ou se há a necessidade de iniciar o processo de reposição dos materiais do estoque, por meio da compra ou de produção própria.

A partir do recebimento dos materiais solicitados, é feita a análise da qualidade, a verificação da quantidade entregue e o registro nos sistemas de materiais da empresa. Em seguida, esses materiais são armazenados de forma adequada para que os níveis de qualidade sejam mantidos e facilitar a entrega. Por fim, a logística consiste na entrega dos materiais solicitados no prazo estipulado e respeitando os padrões de qualidade.

Para que o ciclo de administração dos materiais não seja interrompido, existem modelos de gestão que auxiliam esse processo. Um modelo é a Curva $\mathrm{ABC}$, também conhecida na literatura como Lei de Pareto, em homenagem ao economista Vilfredo Pareto (Pozo, 2010). A partir do estudo, Pareto identificou uma proporção de aproximadamente $80 \%$ e $20 \%$ respectivamente, ou seja, que $80 \%$ da riqueza local estavam concentradas com apenas $20 \%$ da população.

Desde então, este modelo de gestão vem sendo amplamente utilizado em setores onde há a necessidade de tomada de decisão que envolve a análise de um grande volume de dados, como é o caso do gerenciamento de estoques. Assim, de acordo Dias (2010), a partir desta metodologia é possível identificar os itens que merecem atenção, com o objetivo de determinar um tratamento adequado quanto à forma de gestão. Após realizada a ordenação relativa, os itens mais importantes são definidos como (Viana, 2009):

(a) Classe A: grupo de itens mais importantes que devem ser tratados com atenção especial pela administração;

(b) Classe B: grupo de itens em situação intermediária entre as Classes A e C;

(c) Classe C: grupo de itens menos importantes que justificam pouca atenção (Quadro 1).

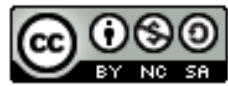


Citação (APA): Siqueira, R. R., Melo, I. A. de A., \& Kato, R. B. (2021). Aplicação da curva abc no gerenciamento de estoque em uma empresa de construção civil. Brazilian Journal of Production Engineering, 7(5), 145-155.

Quadro 1. Reposição do estoque em função da classificação ABC

\begin{tabular}{|c|c|c|c|c|c|}
\hline \multirow{2}{*}{ Classe } & \multicolumn{2}{|c|}{$\begin{array}{c}\text { Média registrada nas } \\
\text { empresas }\end{array}$} & \multirow{2}{*}{$\begin{array}{c}\text { Grau } \\
\text { de } \\
\text { controle }\end{array}$} & \multirow{2}{*}{$\begin{array}{c}\text { Tamanho } \\
\text { de } \\
\text { estoque }\end{array}$} & \multirow{2}{*}{ Procedimentos e constância de reposição } \\
\hline & $\begin{array}{l}\text { Quantidade } \\
\text { (\% de itens) }\end{array}$ & $\begin{array}{c}\text { Valor } \\
(\% \text { de } \mathbf{R} \$)\end{array}$ & & & \\
\hline A & $10-20 \%$ & $70-80 \%$ & Rígido & Baixo & Frequentes, revisões constantes e rigorosas. \\
\hline B & $30-40 \%$ & $15-20 \%$ & Normal & Moderado & Pedidos normais, algum acompanhamento \\
\hline $\mathrm{C}$ & $40-50 \%$ & $5-10 \%$ & Simples & Grande & Pedidos espaçados, suprimento para 1 a 2 anos \\
\hline
\end{tabular}

Fonte: Adaptado de Viana (2009)

Na construção civil, a Curva $\mathrm{ABC}$ vem sendo amplamente utilizada em estudos que envolvem a gestão de estoques. Nesse sentido, Abhilin e Vishak (2017) avaliaram como os sistemas de controle de estoque e o tempo de espera afetam os projetos de construção na cidade Thiruvananthapuram, localizada no sul da Índia. Por meio da curva ABC, os autores verificaram que geralmente a gestão de materiais é realizada de maneira manual nas empresas de construção, o que resulta na falta de controle do consumo exato de materiais e consequentemente na redução do lucro desses empreendimentos.

Zeb et al. (2017) utilizaram a curva ABC para avaliar a importância e o benefício econômico do gerenciamento de estoque para profissionais de construção no Paquistão. Este estudo revelou que cerca de 50-60\% do custo de um projeto está associado ao estoque, e a gestão inadequada dos materiais leva ao excesso de custos e aumenta os atrasos de entrega da construção. Ademais, os autores constataram que o cimento e o aço são os itens de estoques mais significativos, e dessa forma devem ser frequentemente repostos. Enquanto que a areia e pedra estão na Classe $\mathrm{B}$ e $\mathrm{C}$, respectivamente.

Ainda nessa mesma linha de pesquisa, Santos et al. (2020) aplicaram a ferramenta ABC com o intuito de avaliar a gestão de estoque em uma empresa do setor de construção. A partir de entrevistas com o setor do almoxarifado, observações in loco e análise de documentos fornecidos pela empresa, os autores verificaram que 4 materiais em estoque representavam $72,92 \%$ do valor financeiro. Com base nessa informação, Santos et al. (2020) mensuraram os níveis de estoques, dividindo-os em mínimo e mínimo de segurança, e verificaram que apenas um material apresentava uma quantidade ótima no que se refere ao atendimento ao setor produtivo e custos. Logo, concluíram que se a empresa utilizasse os itens da Classe A em níveis mínimo de estoque, poderia haver uma redução nos custos de aquisição de materiais, resultando em um setor mais protegido das variações de saída dos itens para a produção.

\section{METODOLOGIA}

O presente estudo, em termos de classificação em relação a sua natureza, pode ser considerado como pesquisa aplicada. Conforme Silva e Menezes (2005), a pesquisa aplicada tem como finalidade de gerar conhecimento para aplicação prática e é dirigida a problemas específicos. Do ponto de vista da abordagem do problema, é classificada como qualitativa e quantitativa. Richardson (2007) explica que a pesquisa qualitativa leva em consideração o ambiente como uma fonte direta de dados e o pesquisador como uma peça chave. Para os autores Gonçalves e

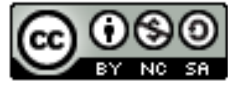



empresa de construção civil. Brazilian Journal of Production Engineering, 7(5), 145-155.

Meirelles (2004) "os dados são representados por métricas quantitativas, tendo como elemento de apoio principal a linguagem matemática”.

A pesquisa também pode ser classificada como estudo de caso, Gil (2008) enfatiza que o estudo de caso vem sendo utilizado com muita frequência pelos pesquisadores científicos. Nos procedimentos técnicos, foram realizadas pesquisas bibliográficas com o intuito de adquirir conhecimento sobre as técnicas inerentes a gestão de estoques. De posse dessas informações foi realizado um estudo de caso, analisando o sistema de gerenciamento de estoques de uma empresa privada que atua no setor da construção civil na cidade de Marabá-PA (Figura 2).

Figura 2. Metodologia da pesquisa

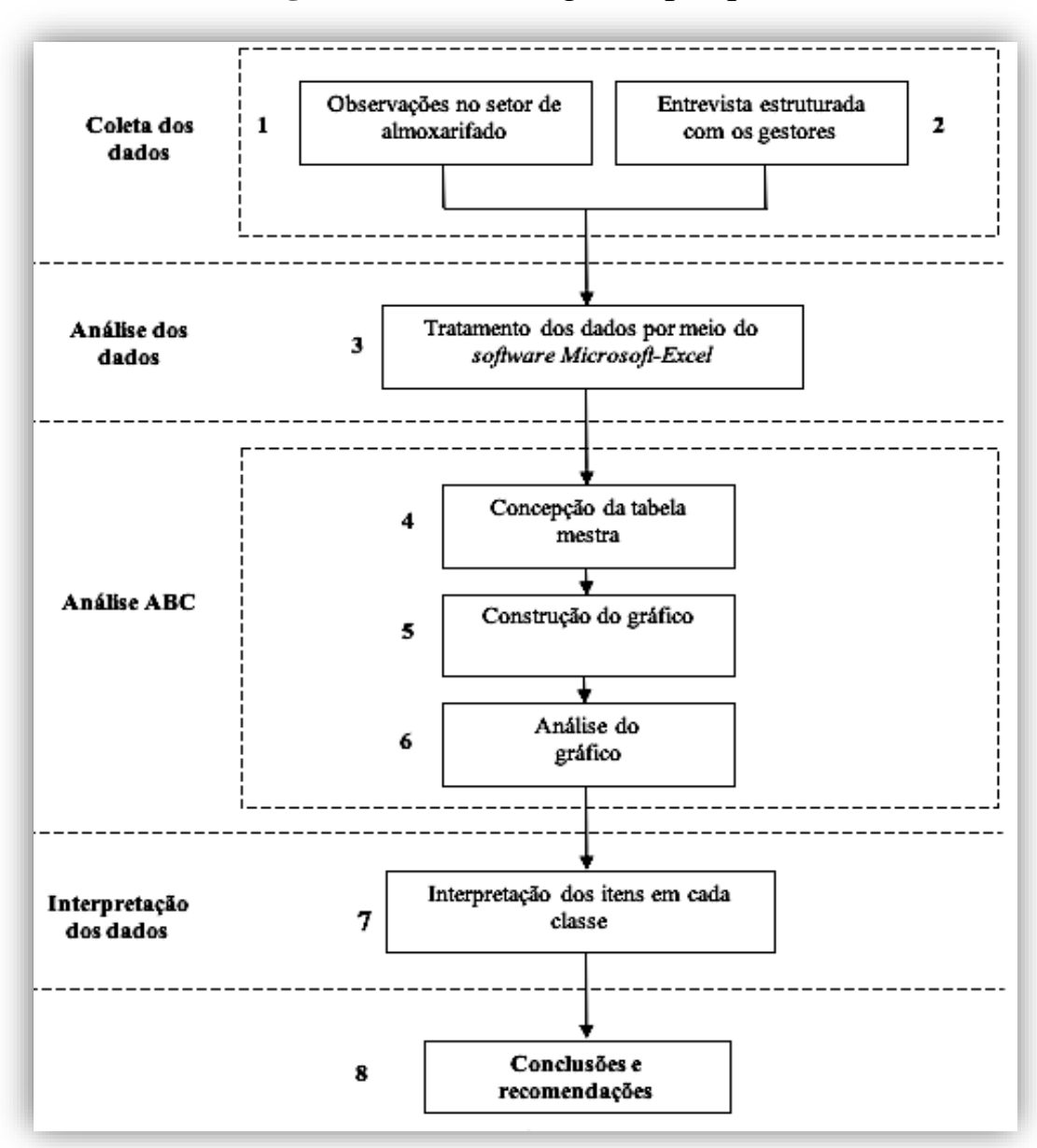

\section{ESTUdO DE CASO}

Fonte: Autores (2021).

\section{Características da empresa}

A empresa que atua no segmento da construção civil é situada na cidade de Marabá, localizado no sudeste do estado do Pará, região Norte do país. A empresa em questão foi fundada na Espanha em 1977 atuando na área da construção civil e consultorias. No Brasil tem sua sede na cidade de Palmas-TO e conta com uma filial na cidade de Marabá-PA.

A empresa atua com serviços de saneamento básico, drenagem, pavimentação e urbanização. Tendo como campo de atuação construção de rodovias, obras de saneamento básico, barragens, portos, aeroportos, obras industrias e habitacionais. Nessa perspectiva, a empresa tem como 

empresa de construção civil. Brazilian Journal of Production Engineering, 7(5), 145-155.

objetivo proporcionar soluções de excelência na procura pela satisfação dos seus clientes, colaboradores e acionistas.

\section{RESUltados E DiscuSSÃo}

Após serem feitas análises em todo o processo de gerenciamento de estoques da empresa de construção civil, foi possível constatar grandes problemas enfrentados pelo gestor da organização para a tomada de decisão, devido ao não uso ou desconhecimento de ferramentas de apoio.

Atualmente na empresa, o processo de compra de produtos é feito utilizando-se de observações empíricas. Sendo assim, o setor de compras solicita dos fornecedores os devidos itens que estão em menor quantidade no estoque, sem observar qual a importância desse item para o andamento da obra. Quando os itens adquiridos chegam, vão para o almoxarifado, para conferência e armazenamento.

Com relação à saída de produtos, o processo é feito através de requisições. O operador de almoxarifado recebe as requisições dos engenheiros ou mestre de obra, fornecem os produtos e anexam os requerimentos para posterior saída no sistema. Como não é feito um acompanhamento diário e rigoroso da quantidade de itens que saiu e o que tem em estoque, na maioria das vezes há incompatibilidade entre o físico e o software, em relação à quantidade e os custos reais de saída dos produtos.

Como foram descritos anteriormente, o gerenciamento de estoques na empresa é feito por meio de observações, deste modo, o uso de ferramentas de apoio torna-se importante para criar vantagens competitivas em relação aos concorrentes, trazendo consigo informações concretas para a tomada de decisão. Nesta situação, foi aplicado a ferramenta de Classificação ABC na tentativa de sanar determinados problemas apresentados na empresa.

\section{Classificação ABC e Gráfico de Pareto}

A empresa em questão apresenta uma grande variedade de materiais utilizados na sua obra de ampliação da rede de água e esgoto, e não possuía controle de quais dos itens tinha a maior representatividade nos seus custos, ou quais itens são considerados de maior importância. Para que fosse possível observar o comportamento da demanda dos itens, foi construído nas Tabelas a seguir, com a classificação ABC dos itens utilizados (Tabela 1).

Tabela 1. Classificação Curva A dos materiais

\begin{tabular}{|c|c|c|c|c|c|c|c|c|}
\hline Código & Descrição & Qtd. & $\begin{array}{l}\text { Valor Uni. } \\
\text { (R\$) }\end{array}$ & $\begin{array}{c}\text { Custo Total } \\
\text { (R\$) }\end{array}$ & $\%$ & $\begin{array}{c}\% \\
\text { Acumulado }\end{array}$ & $\begin{array}{c}\% \\
\text { Itens }\end{array}$ & Classe \\
\hline A1 & $\begin{array}{c}\text { Colar De } \\
\text { Tomada Pvc } \\
\text { C/Travas } 60 \\
\text { Mmx } 1 / 2\end{array}$ & 3350 & $\mathrm{R} \$ 69,21$ & $\begin{array}{c}\mathrm{R} \$ \\
231.853,50\end{array}$ & $30,49 \%$ & $30,49 \%$ & $2 \%$ & \\
\hline A2 & $\begin{array}{c}\text { Medidor } \\
\text { Aquarius V4 Qn } \\
\text { 1,5 B 20/115 }\end{array}$ & 4000 & $\mathrm{R} \$ 41,76$ & $\begin{array}{c}\mathrm{R} \$ \\
167.040,00\end{array}$ & $21,97 \%$ & $52,46 \%$ & $3 \%$ & $\mathbf{A}$ \\
\hline $\mathbf{A 3}$ & $\begin{array}{l}\text { Medidor Vmf } \\
\text { Dn } 500 \mathrm{Mm}+ \\
\text { Ind. Img } 10\end{array}$ & 2 & $\mathrm{R} \$ 39.870,00$ & $\begin{array}{c}\mathrm{R} \$ \\
79.740,00\end{array}$ & $10,49 \%$ & $62,94 \%$ & $5 \%$ & \\
\hline A4 & $\begin{array}{l}\text { Caixa Dn } 3 / 4 \\
\text { Termoplastica }\end{array}$ & 4000 & $R \$ 16,00$ & $\begin{array}{c}\mathrm{R} \$ \\
64.000,00\end{array}$ & $8,42 \%$ & $71,36 \%$ & $7 \%$ & \\
\hline
\end{tabular}

Fonte: Autores (2021). 
- 151 -

Citação (APA): Siqueira, R. R., Melo, I. A. de A., \& Kato, R. B. (2021). Aplicação da curva abc no gerenciamento de estoque em uma empresa de construção civil. Brazilian Journal of Production Engineering, 7(5), 145-155.

Tabela 1.1. Classificação Curva B dos materiais

\begin{tabular}{|c|c|c|c|c|c|c|c|}
\hline A5 & $\begin{array}{c}\text { Cjto Conexoes De 3/4 } \\
\text { Em Pvc Branco }\end{array}$ & 4000 & $\mathrm{R} \$ 15,00$ & $\begin{array}{c}\mathrm{R} \$ \\
60.000,00\end{array}$ & $7,89 \%$ & $79,25 \%$ & $9 \%$ \\
\hline A6 & $\begin{array}{l}\text { Tubo Fofo Ponta Flange } \\
\text { Dn } 100 \text { Pn10 - L=1500 }\end{array}$ & 14 & $\mathrm{R} \$ 1.019,00$ & $\begin{array}{c}\mathrm{R} \$ \\
14.266,00\end{array}$ & $1,88 \%$ & $81,13 \%$ & $10 \%$ \\
\hline A7 & $\begin{array}{c}\text { Luva Fofo Junta } \\
\text { Mecanica Dn } 700 \mathrm{Mm}\end{array}$ & 2 & $\mathrm{R} \$ 5.280,00$ & $\begin{array}{c}\mathrm{R} \$ \\
10.560,00\end{array}$ & $1,39 \%$ & $82,52 \%$ & $12 \%$ \\
\hline A8 & $\begin{array}{c}\text { Colar De Tomada Dn } \\
150 \mathrm{Mm} 1 / 2\end{array}$ & 623 & $\mathrm{R} \$ 14,68$ & $\mathrm{R} \$ 9.145,64$ & $1,20 \%$ & $83,72 \%$ & $14 \%$ \\
\hline A9 & $\begin{array}{l}\text { Tubo Fofo Ponta Bolsa } \\
\text { Dn } 700 \mathrm{Mm} \mathrm{L}=5,80 \mathrm{M}\end{array}$ & 1 & $\mathrm{R} \$ 7.480,00$ & $\mathrm{R} \$ 7.480,00$ & $0,98 \%$ & $84,70 \%$ & $16 \%$ \\
\hline A10 & $\begin{array}{l}\text { Te Fofo Bolsa Flange } \\
\text { Dn } 500 \text { X } 100 \mathrm{Mm}\end{array}$ & 4 & $\mathrm{R} \$ 1.840,00$ & $\mathrm{R} \$ 7.360,00$ & $0,97 \%$ & $85,67 \%$ & $17 \%$ \\
\hline A11 & $\begin{array}{c}\text { Luva De Correr C/Bolsa } \\
\text { Jgs Dn } 400 \mathrm{Mm}\end{array}$ & 13 & $\mathrm{R} \$ 549,44$ & $\mathrm{R} \$ 7.142,72$ & $0,94 \%$ & $86,61 \%$ & $19 \%$ \\
\hline A12 & $\begin{array}{c}\text { Cruzeta C/Bolsa Jgs Dn } \\
150 \times 100 \mathrm{Mm}\end{array}$ & 28 & $\mathrm{R} \$ 233,90$ & $\mathrm{R} \$ 6.549,20$ & $0,86 \%$ & $87,47 \%$ & $21 \%$ \\
\hline A13 & $\begin{array}{c}\text { Tubo Fofo Ponta Flange } \\
\text { Pn } 16 \mathrm{Dn} 100 \mathrm{Mm} X \\
5,80 \mathrm{Mm}\end{array}$ & 5 & $\mathrm{R} \$ 1.261,40$ & $\mathrm{R} \$ 6.307,00$ & $0,83 \%$ & $88,30 \%$ & $22 \%$ \\
\hline A14 & $\begin{array}{c}\text { Te Bbb Jgs Dn } 150 \mathrm{X} \\
100 \mathrm{Mm}\end{array}$ & 35 & $\mathrm{R} \$ 178,12$ & $\mathrm{R} \$ 6.234,20$ & $0,82 \%$ & $89,12 \%$ & $24 \%$ \\
\hline A15 & $\begin{array}{l}\text { Tubo Fofo Flangeado } \\
\text { Dn } 500 \mathrm{Mm} \text { X 5,80 M }\end{array}$ & 1 & $\mathrm{R} \$ 5.910,20$ & $\mathrm{R} \$ 5.910,20$ & $0,78 \%$ & $89,90 \%$ & $26 \%$ \\
\hline A16 & $\begin{array}{c}\text { Colar De Tomada Dn } \\
250 \mathrm{Mm} 1 / 2\end{array}$ & 192 & $\mathrm{R} \$ 22,90$ & $\mathrm{R} \$ 4.396,80$ & $0,58 \%$ & $90,47 \%$ & $28 \%$ \\
\hline A17 & $\begin{array}{c}\text { Redução Pb Jgs Dn } 150 \\
\text { X } 100 \text { Mm }\end{array}$ & 41 & $\mathrm{R} \$ 96,79$ & $\mathrm{R} \$ 3.968,39$ & $0,52 \%$ & $91,00 \%$ & $29 \%$ \\
\hline A18 & $\begin{array}{c}\text { CURVA BB JGS } 90^{\circ} \mathrm{X} \\
400 \mathrm{MM}\end{array}$ & 4 & $\mathrm{R} \$ 961,51$ & $\mathrm{R} \$ 3.846,04$ & $0,51 \%$ & $91,50 \%$ & $31 \%$ \\
\hline
\end{tabular}

Fonte: Autores (2021).

Tabela 1.2. Classificação Curva $\mathrm{C}$ dos materiais

\begin{tabular}{|c|c|c|c|c|c|c|c|}
\hline A19 & $\begin{array}{l}\text { Cruzeta De Fd Bolsas Je } \\
\text { Dn } 400 \text { X } 100 \mathrm{Mm}\end{array}$ & 4 & $\mathbf{R} \$ \mathbf{8 8 0 , 0 0}$ & $\mathbf{R} \$ \mathbf{3 . 5 2 0 , 0 0}$ & $0,46 \%$ & $91,97 \%$ & $33 \%$ \\
\hline A20 & $\begin{array}{c}\text { Te Bbb Jgs Dn } 200 \text { X } 100 \\
\text { Mm }\end{array}$ & 15 & $\mathrm{R} \$ 227,45$ & $\mathrm{R} \$ 3.411,75$ & $0,45 \%$ & $92,41 \%$ & $34 \%$ \\
\hline A21 & $\begin{array}{c}\text { Colar De Tomada Ff P/Ff } \\
\text { Dn } 150 \times 1 / 2\end{array}$ & 150 & $\mathrm{R} \$ 22,10$ & $\mathrm{R} \$ 3.315,00$ & $0,44 \%$ & $92,85 \%$ & $36 \%$ \\
\hline A22 & $\underset{\mathrm{C}}{\mathrm{CURVA}} \underset{\mathrm{N}}{500 \mathrm{MM}} 45$ & 4 & $\mathrm{R} \$ 807,00$ & $\mathrm{R} \$ 3.228,00$ & $0,42 \%$ & $93,27 \%$ & $38 \%$ \\
\hline A23 & $\begin{array}{c}\text { Cruzeta C/Bolsa Jgs Dn } \\
400 \text { X } 200 \mathrm{Mm}\end{array}$ & 3 & $\mathrm{R} \$ 952,36$ & $\mathrm{R} \$ 2.857,08$ & $0,38 \%$ & $93,65 \%$ & $40 \%$ \\
\hline A24 & $\begin{array}{l}\text { Colar De Tomada Pvc } \\
\text { C/Travas } 110 \mathrm{Mmx} 1 / 2\end{array}$ & 200 & $\mathrm{R} \$ 11,64$ & $\mathrm{R} \$ 2.328,00$ & $0,31 \%$ & $93,96 \%$ & $41 \%$ \\
\hline A25 & $\begin{array}{c}\text { Redução Pvc Je Pb De } \\
\text { 110x60 Pba }\end{array}$ & 166 & $\mathrm{R} \$ 13,12$ & $\mathrm{R} \$ 2.177,92$ & $0,29 \%$ & $94,24 \%$ & $43 \%$ \\
\hline A26 & $\begin{array}{c}\text { Cruzeta C/Bolsa Jgs Dn } \\
150 \times 150 \mathrm{Mm}\end{array}$ & 8 & $\mathrm{R} \$ 266,16$ & $\mathrm{R} \$ 2.129,28$ & $0,28 \%$ & $94,52 \%$ & $45 \%$ \\
\hline A27 & Te Dn 250 X $100 \mathrm{Mm}$ & 7 & $\mathrm{R} \$ 304,00$ & $\mathrm{R} \$ 2.128,00$ & $0,28 \%$ & $94,80 \%$ & $47 \%$ \\
\hline A28 & $\begin{array}{c}\text { Te Bbb Jgs Dn } 400 \text { X } 100 \\
\text { Mm }\end{array}$ & 3 & $\mathrm{R} \$ 705,11$ & $\mathrm{R} \$ 2.115,33$ & $0,28 \%$ & $95,08 \%$ & $48 \%$ \\
\hline A29 & $\begin{array}{c}\text { Redução Fofo Ponta } \\
\text { Bolsa Jgs Dn } 700 \text { X } 500 \\
\text { Mm }\end{array}$ & 1 & $\mathrm{R} \$ 2.107,00$ & $\mathrm{R} \$ 2.107,00$ & $0,28 \%$ & $95,36 \%$ & $50 \%$ \\
\hline A30 & $\begin{array}{c}\text { Te Bbb Jgs Dn } 150 \text { X } 150 \\
\text { Mm }\end{array}$ & 12 & $\mathrm{R} \$ 165,34$ & $\mathrm{R} \$ 1.984,08$ & $0,26 \%$ & $95,62 \%$ & $52 \%$ \\
\hline A31 & $\begin{array}{c}\text { Anel De Borracha Pba Dn } \\
100 \text { De } 110 \mathrm{Mm}\end{array}$ & 427 & $\mathrm{R} \$ 4,49$ & $\mathrm{R} \$ 1.917,23$ & $0,25 \%$ & $95,87 \%$ & $53 \%$ \\
\hline A32 & $\begin{array}{l}\text { Tubo Coletor (Ocre) Jei } \\
\text { Dn } 150\end{array}$ & 20 & $\mathrm{R} \$ 92,63$ & $\mathrm{R} \$ 1.852,60$ & $0,24 \%$ & $96,11 \%$ & $55 \%$ \\
\hline A33 & $\begin{array}{c}\text { Cruzeta C/Bolsa Jgs Dn } \\
250 \text { X } 100 \mathrm{Mm}\end{array}$ & 5 & $\mathrm{R} \$ 367,22$ & $\mathrm{R} \$ 1.836,10$ & $0,24 \%$ & $96,36 \%$ & $57 \%$ \\
\hline
\end{tabular}


- 152 -

Citação (APA): Siqueira, R. R., Melo, I. A. de A., \& Kato, R. B. (2021). Aplicação da curva abc no gerenciamento de estoque em uma empresa de construção civil. Brazilian Journal of Production Engineering, 7(5), 145-155.

\begin{tabular}{|c|c|c|c|c|c|c|c|}
\hline A34 & $\begin{array}{c}\text { Te Bb Jgs Dn } 500 \text { X } 500 \\
\text { Mm }\end{array}$ & 1 & $\mathrm{R} \$ 1.827,34$ & $\mathrm{R} \$ 1.827,34$ & $0,24 \%$ & $96,60 \%$ & $59 \%$ \\
\hline A35 & $\begin{array}{c}\text { Colar De Tomada Dn } 200 \\
\text { Mm } 1 / 2\end{array}$ & 106 & $\mathrm{R} \$ 17,08$ & $\mathrm{R} \$ 1.810,48$ & $0,24 \%$ & $96,83 \%$ & $60 \%$ \\
\hline A36 & $\begin{array}{l}\text { Cruzeta Bb Jgs Dn } 200 \\
\text { Mm X } 100 \mathrm{Mm}\end{array}$ & 7 & $\mathrm{R} \$ 250,03$ & $\mathrm{R} \$ 1.750,21$ & $0,23 \%$ & $97,06 \%$ & $62 \%$ \\
\hline A37 & $\begin{array}{c}\text { CURVA BB JGS } 90^{\circ} \mathrm{X} \\
500 \mathrm{MM}\end{array}$ & 1 & $\mathrm{R} \$ 1.748,32$ & $\mathrm{R} \$ 1.748,32$ & $0,23 \%$ & $97,29 \%$ & $64 \%$ \\
\hline A38 & $\begin{array}{l}\text { Luva De Correr C/Bolsa } \\
\text { Jgs Dn } 200 \mathrm{Mm}\end{array}$ & 11 & $\mathrm{R} \$ 141,95$ & $\mathrm{R} \$ 1.561,45$ & $0,21 \%$ & $97,50 \%$ & $66 \%$ \\
\hline A39 & $\begin{array}{l}\text { Luva De Correr C/Bolsa } \\
\text { Jgs Dn } 250 \mathrm{Mm}\end{array}$ & 6 & $\mathrm{R} \$ 239,12$ & $\mathrm{R} \$ 1.434,72$ & $0,19 \%$ & $97,69 \%$ & $67 \%$ \\
\hline A40 & $\begin{array}{l}\text { Anel De Borracha Pba Dn } \\
50\end{array}$ & 1127 & $\mathrm{R} \$ 1,14$ & $\mathrm{R} \$ 1.284,78$ & $0,17 \%$ & $97,86 \%$ & $69 \%$ \\
\hline A41 & $\begin{array}{c}\text { Redução Pb Jgs Dn } 500 \text { X } \\
400 \mathrm{Mm}\end{array}$ & 2 & $\mathrm{R} \$ 622,28$ & $\mathrm{R} \$ 1.244,56$ & $0,16 \%$ & $98,02 \%$ & $71 \%$ \\
\hline A42 & $\begin{array}{c}\text { Luva Correr Pvc Je } \\
\text { De110 Pba }\end{array}$ & 55 & $\mathrm{R} \$ 20,73$ & $\mathrm{R} \$ 1.140,15$ & $0,15 \%$ & $98,17 \%$ & $72 \%$ \\
\hline A43 & $\begin{array}{c}\text { Redução Pb Jgs Dn } 400 \text { X } \\
250 \mathrm{Mm}\end{array}$ & 2 & $\mathrm{R} \$ 554,02$ & $\mathrm{R} \$ 1.108,04$ & $0,15 \%$ & $98,32 \%$ & $74 \%$ \\
\hline A44 & $\begin{array}{l}\text { Tubo Coletor (Ocre) Jei } \\
\text { Dn } 100\end{array}$ & 25 & $\mathrm{R} \$ 43,66$ & $\mathrm{R} \$ 1.091,50$ & $0,14 \%$ & $98,46 \%$ & $76 \%$ \\
\hline A45 & $\begin{array}{l}\text { Cruzeta C/Bolsa Jgs Dn } \\
250 \times 250 \mathrm{Mm}\end{array}$ & 2 & $\mathrm{R} \$ 532,89$ & $\mathrm{R} \$ 1.065,78$ & $0,14 \%$ & $98,60 \%$ & $78 \%$ \\
\hline A46 & $\begin{array}{c}\text { Te Bbb Jgs Dn } 400 \text { X } 400 \\
\text { Mm }\end{array}$ & 1 & $\mathrm{R} \$ 1.053,09$ & $\mathrm{R} \$ 1.053,09$ & $0,14 \%$ & $98,74 \%$ & $79 \%$ \\
\hline A47 & $\begin{array}{c}\text { Redução Pvc Je Pb De } \\
\text { 110x85 Pba }\end{array}$ & 66 & $\mathrm{R} \$ 15,29$ & $\mathrm{R} \$ 1.009,14$ & $0,13 \%$ & $98,87 \%$ & $81 \%$ \\
\hline A48 & $\begin{array}{l}\text { Luva De Correr C/Bolsa } \\
\text { Jgs Dn } 150 \mathrm{Mm}\end{array}$ & 10 & $\mathrm{R} \$ 100,82$ & $\mathrm{R} \$ 1.008,20$ & $0,13 \%$ & $99,00 \%$ & $83 \%$ \\
\hline A49 & $\begin{array}{c}\text { Luva Correr Pvc Je } 60 \\
\text { Pba }\end{array}$ & 190 & $\mathrm{R} \$ 5,02$ & $\mathrm{R} \$ 953,80$ & $0,13 \%$ & $99,13 \%$ & $84 \%$ \\
\hline A50 & $\begin{array}{c}\text { Cruzeta Dn } 400 \text { X } 100 \\
\text { Mm }\end{array}$ & 1 & $\mathrm{R} \$ 924,00$ & $\mathrm{R} \$ 924,00$ & $0,12 \%$ & $99,25 \%$ & $86 \%$ \\
\hline A51 & Anel Je Dn 75/De 85 Pba & 346 & $\mathrm{R} \$ 2,58$ & $\mathrm{R} \$ 892,68$ & $0,12 \%$ & $99,37 \%$ & $88 \%$ \\
\hline A52 & $\begin{array}{c}\text { Redução Pb Jgs Dn } 250 \text { X } \\
150 \mathrm{Mm}\end{array}$ & 5 & $\mathrm{R} \$ 166,17$ & $\mathrm{R} \$ 830,85$ & $0,11 \%$ & $99,48 \%$ & $90 \%$ \\
\hline A53 & $\begin{array}{c}\text { Redução Pb Jgs Dn } 200 \text { X } \\
150 \mathrm{Mm}\end{array}$ & 5 & $\mathrm{R} \$ 141,15$ & $\mathrm{R} \$ 705,75$ & $0,09 \%$ & $99,57 \%$ & $91 \%$ \\
\hline A54 & $\begin{array}{l}\text { Anel De Borracha Pba Dn } \\
7585 \mathrm{Mm}\end{array}$ & 163 & $\mathrm{R} \$ 4,19$ & $\mathrm{R} \$ 682,97$ & $0,09 \%$ & $99,66 \%$ & $93 \%$ \\
\hline A55 & $\begin{array}{c}\text { Te Pvc Je Bbb De 110x60 } \\
\text { Pba }\end{array}$ & 22 & $\mathrm{R} \$ 30,25$ & $\mathrm{R} \$ 665,50$ & $0,09 \%$ & $99,75 \%$ & $95 \%$ \\
\hline A56 & $\begin{array}{c}\text { Cruzeta C/Bolsa Jgs Dn } \\
200 \times 150 \mathrm{Mm}\end{array}$ & 2 & $\mathrm{R} \$ 330,69$ & $\mathrm{R} \$ 661,38$ & $0,09 \%$ & $99,83 \%$ & $97 \%$ \\
\hline A57 & $\begin{array}{c}\text { Redução Pvc Pba Pb De } \\
85 \text { X Dn } 60 \mathrm{Mm}\end{array}$ & 63 & $\mathrm{R} \$ 10,41$ & $\mathrm{R} \$ 655,83$ & $0,09 \%$ & $99,92 \%$ & $98 \%$ \\
\hline \multirow[t]{2}{*}{ A58 } & $\begin{array}{l}\text { Curva Pvc Pba Je } \mathrm{Pb} 22^{\circ} \\
\text { Dn } 75 \mathrm{De} 85 \mathrm{Mm}\end{array}$ & 20 & $\mathrm{R} \$ 29,98$ & $\mathrm{R} \$ 599,60$ & $0,08 \%$ & $100,00 \%$ & $100 \%$ \\
\hline & & & SOMA & $\begin{array}{c}R \$ \\
760.417,18\end{array}$ & & & \\
\hline
\end{tabular}

Fonte: Autores (2021).

Os dados utilizados são referentes ao ano de 2019 e foram fornecidos pela empresa. Utilizando o software Microsoft Excel foram calculadas as quantidades médias mensais dos produtos existentes. Para a construção da tabela mestra foram utilizados somente os 58 produtos que apresentavam maior quantidade média no período. Deste modo, com a identificação dos produtos na classe A, B e C, foi possível a construção da curva ABC (Figura 3).

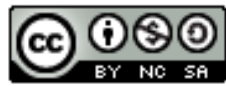


Citação (APA): Siqueira, R. R., Melo, I. A. de A., \& Kato, R. B. (2021). Aplicação da curva abc no gerenciamento de estoque em uma empresa de construção civil. Brazilian Journal of Production Engineering, 7(5), 145-155.

Figura 3. Gráfico de Pareto

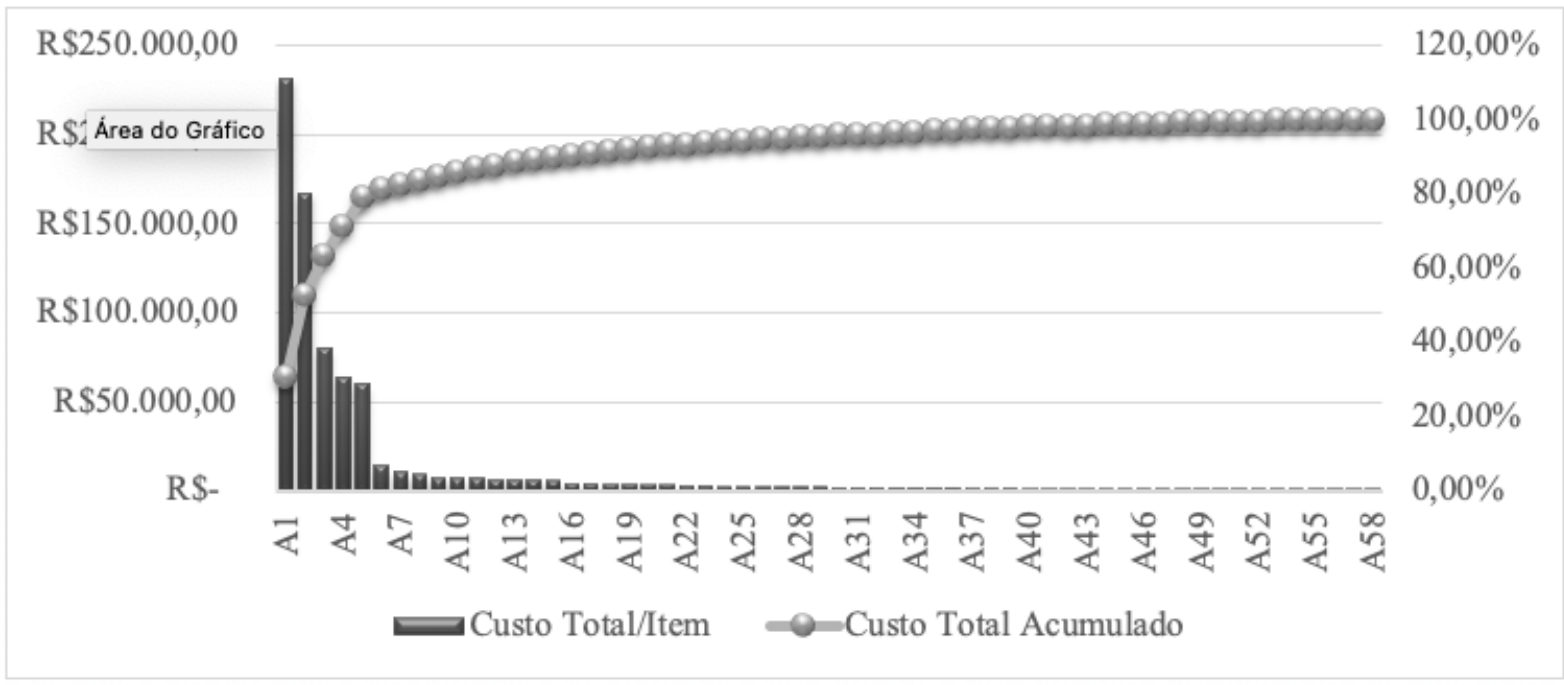

Fonte: Autores (2021).

A Tabela 1 e a Figura 3 demonstram que 7\% dos itens representam 71,36\% do custo total gerado no ano de 2019 para a empresa em estudo. Isso permite dizer que uma quantidade pequena de produtos representa mais da metade dos custos gerados. Em virtude deste fato, esses produtos foram classificados como A. Para classificação B, 24\% dos itens representam 20,14\% do custo total. Finalmente, $69 \%$ dos itens representam $8,50 \%$ do total do mencionado custo, sendo classificado como itens de classe $\mathrm{C}$.

Os resultados obtidos contrariam a teoria proposta por Vilfredo Pareto, de estoques no sistema $80 / 20$, e que itens da classe A devem possuir estoques baixos, enquanto que itens da classe $\mathrm{C}$ devem ter estoques altos. Analisando-se a Tabela 1, verifica-se que os itens classificados como A, possuem tamanho de estoque alto, com 11.352 dos 19.552 materiais presentes no almoxarifado. Por outro lado, os itens classificados como C correspondem a apenas 3.237 dos materiais em estoque. Um dos motivos que levou a esses resultados foi a falta de utilização de ferramentas de gestão para quantificar e qualificar os itens que devem ser comprados. Logo, os materiais em estoque foram adquiridos baseados apenas no conhecimento empírico e sem nenhum critério para a escolha dos itens e da quantidade necessária para o bom funcionamento da empresa.

\section{CONCLUSÃo}

Vago et al. (2013) confirmam a importância de ter uma boa gestão de estoque na administração eficiente dos materiais nas organizações, visto isso, a escolha de uma ferramenta que melhor se encaixe nas necessidades da empresa torna se essencial para solucionar diferentes problemas que possam vim a enfrentar.

Nesse sentido, conforme o estudo feito em relação ao almoxarifado na Organização do ramo da construção civil na cidade de Marabá-PA, identificou-se a necessidade de utilizar técnicas de análise dos estoques para o andamento das movimentações dos materiais dentro do setor. Logo, optou-se pelo o uso da ferramenta da curva ABC.

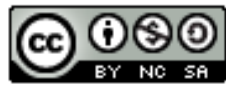



empresa de construção civil. Brazilian Journal of Production Engineering, 7(5), 145-155.

A afirmação da utilização desta ferramenta deu-se primeiramente pela análise do setor de almoxarifado e posteriormente uma entrevista com a gestor da Organização e assim aplicou se a curva $\mathrm{ABC}$. Uma observação da curva $\mathrm{ABC}$, proporcionou diagnosticar os itens de maior importância financeira para a empresa e quais os itens que precisariam ter mais atenção. Em suma, com o uso da ferramenta $\mathrm{ABC}$, foi possível analisar que os procedimentos existentes de gerenciamento de inventário na organização eram precários, e as informações obtidas não condiziam com o real.

Depois de descritas as atividades principais que são desenvolvidas em um almoxarifado, identificou-se, por meio de entrevista com a gestora da Organização, como são desenvolvidas essas atividades. Assim, desenvolveu se uma análise com a curva ABC para visualizar melhor o mapa do almoxarifado com relação a todos os materiais. Como descrito por diferentes autores, com base no levantamento dos dados, chegou-se perto da equação 80/20 quanto à proporção da movimentação dos itens do estoque em relação ao total de itens, pois foi identificado que aproximadamente $78 \%$ da movimentação desses itens correspondem a $13 \%$ do seu total. Assim, ficou demonstrado que, para controlar, em média, 78\% do estoque, basta administrar melhor $13 \%$ dos itens. Por meio da curva $\mathrm{ABC}$, foi possível visualizar a situação atual do estoque, conhecendo a importância de cada material e a quantidade de cada classe.

Diante disso, através da aplicação da referida ferramenta, a real situação e as futuras melhorias advindas com a implantação da mesma, foi observada por meio de tabelas, gráficos e informações geradas, pontuando assim novos critérios de atuação concernente as atividades de gestão outrora praticada, melhorando as tomadas de decisão dos gerentes do setor, aumentando assim a eficiência no gerenciamento.

Em resumo o uso da ferramenta $\mathrm{ABC}$ na empresa de construção civil, permitiu a gestão em conhecer as verdadeiras necessidades. Agora, é possível planejar o suprimento de estoques focando os itens que representam o maior consumo e o menor, trazendo um equilíbrio entre necessidades e disponibilidade de recursos.

\section{REFERÊNCIAS}

Abhilin, G. B., \& Vishak, M. S. (2017). Effective Material Logistics in Construction Industries. International Journal of Science and Research, 6(3), 910-913.

Dias, M. A. P. (2010). Administração de materiais: uma abordagem logística. 5 ed. São Paulo: Atlas.

Gil , A. C. (2008). Como elaborar projetos de pesquisa. 4. Ed. São Paulo: Atlas, 2008.

Gonçalves, C. A., \& Meirelles, A. M. (2004). Projetos e relatórios de pesquisa em administração. São Paulo: Atlas.

Instituto Brasileiro de Geografia e Estatística (2019). Produto Interno Bruto - 2019. Recuperado de https://www.ibge.gov.br/?_ga=2.49988270.926593430.1612981068$\underline{282461629.1612197871}$

Martins, P. G., \& Laugeni, F. P. (2009). Administração da Produção e Operações. São Paulo: Saraiva. 
Pozo, H. (2010). Administração de Recursos Materiais e Patrimoniais, uma abordagem Logística. 6. ed. São Paulo: Atlas.

Richardson, R. J. (2007). Pesquisa Social: métodos e técnicas. 3. ed. São Paulo: Atlas.

Santos, L. R. V., Sant'anna, C. H. M., Sant'anna, A. M., Guimarães, D. S., Jr., \& Melo, F. J. C. (2020). Aplicação de ferramentas da gestão de estoque com foco na logística dos materiais em uma indústria de construção civil. Revista OPARA, 10(1), 55-78

Silva, E. L., \& Menezes, E. M. (2005). Metodologia da pesquisa e elaboração de dissertação. 4. ed. Florianópolis.

Szajubok, N. K., Alencar, L. H., \& Almeida, A. T. D. (2006). Modelo de gerenciamento de materiais na construção civil utilizando avaliação multicritério. Produção, 16, 303318. http://dx.doi.org/10.1590/S0103-65132006000200010

Vago, F. R. M., Veloso, C., do Couto, J. M., Lara, J. E., Fagundes, A. F. A., \& de Oliveira Sampaio, D. (2013). A importância do gerenciamento de estoque por meio da ferramenta curva ABC. Revista Sociais e Humanas, 26(3), 638-655.

Viana, J. J. (2009). Administração de materiais: um enfoque prático. 1 ed. São Paulo: Atlas.

Zeb, A., Khan, D., Sajid, M., \& Khattak, S. B. (2017). Inventory analysis of construction project. In: International Conference on Industrial Engineering and Management Applications, 1, Jamshoro. Anais... Jamshoro: Mehran University of Engineering and Technology, p. 68-74. 